\title{
Resultados auditivos de estapedostomías en platinas complicadas
}

\section{Hearing results on stapedostomies in complicated platens}

\author{
Carlos Stott $\mathrm{C}^{1}$, Michel Royer $\mathrm{F}^{2}$, Rubén Olmedo $\mathrm{O}^{3}$, Cristian Martínez $\mathrm{S}^{3}$, \\ Constanza Valdés $\mathrm{P}^{4}$, Lorena Ortúzar $\mathrm{B}^{4}$.
}

\begin{abstract}
RESUMEN
La estapedostomía es una alternativa de tratamiento quirúrgico de la otoesclerosis altamente eficaz y con resultados estables a largo plazo. Uno de los factores que puede condicionar una peor ganancia auditiva postoperatoria es la presencia de alteraciones significativas en la platina del estribo. Se incluyen en este estudio pacientes con otoesclerosis que fueron sometidos a estapedostomía entre los años 2000 a 2005 y en los cuales se constató la presencia de platina obliterada o flotante. Se compararon los valores auditivos pre y postoperatorios con un grupo de referencia constituido por pacientes sometidos a estapedostomía en el mismo período con platina azul. Se incluyeron 14 pacientes, sin diferencias en edad y género con respecto a los controles. No existieron diferencias estadísticamente significativas entre los grupos en cuanto a umbrales de vía ósea, aérea ni diferencia óseo-aérea. No se presentaron complicaciones en esta serie. Estos resultados demuestran que una técnica quirúrgica apropiada, junto a la experiencia del equipo quirúrgico hacen de la estapedostomía una alternativa exitosa en este tipo de pacientes.
\end{abstract}

Palabras clave: Otoesclerosis, Estapedostomía, platina obliterada, platina flotante

\section{SUMMARY}

Stapedostomy is a highly efficient alternative surgical treatment for otosclerosis and with stable results in the long term. One of the factors that may condition a lower postoperatory auditive increase is the presence of significant alterations in the stirrup bone platen. Included in this study are patients with otosclerosis who were subject to

\footnotetext{
${ }^{1}$ Médico Otorrinolaringólogo, Hospital Cínico de la Universidad de Chile.

${ }^{2}$ Médico Residente de Otorrinolaringología, Hospital Cínico de la Universidad de Chile.

${ }^{3}$ Tecnólogo Médico, Hospital Cínico de la Universidad de Chile.

${ }^{4}$ Interna de Medicina, Facultad de Medicina, Universidad de Chile.
} 
stapedostomy between years 2002 and 2005 and in which the presence of obliterated or floating platen is found. Pre and post-operatory values were compared with a reference group constituted by patients subjected to stapedostomy in the same period with blue platen. 14 patients were included, without age or gender differences in connection to controls. There were no significant statistical differences between the groups in connection to bone path, air path, or bone-air difference. No complications appeared in this series. These results show that an adequate surgical technique, together with the experience of the surgical team make stapedostomy a successful alternative in this type of patients.

Key words: Atosclerosis, Stapedostomy, obliterated platen, floating platen.

\section{INTRODUCCION}

La otoesclerosis es una enfermedad de causa desconocida, con una base genética de transmisión autosómica dominante de baja penetrancia y expresividad variable ${ }^{1}$, en la cual participarían otros factores en su desarrollo como enzimáticos ${ }^{2,3}$, inmunológicos ${ }^{4,5}$ e infecciones virales ${ }^{6,7}$. Afecta en forma exclusiva el hueso temporal humano, específicamente la cápsula ótica, en la que en una primera etapa se observa mayor vascularización y reabsorción del hueso, fenómeno denominado otoespongiosis. Posteriormente predomina la neoformación y remodelación ósea (esclerosis) con depósito de tejido óseo anormal. Esta actividad puede presentarse en uno o múltiples focos, e incluso dentro de un mismo foco pueden coexistir diversas fases del proceso ${ }^{8}$.

La presentación clínica característica de esta enfermedad es una hipoacusia de conducción, que se produce cuando el foco de otoesclerosis compromete la platina del estribo y provoca la fijación de ésta. También puede presentar un componente sensorioneural, histológicamente relacionado con una extensión medial del foco, hacia la cóclea 9 .

Una de las alternativas de tratamiento quirúrgico es la estapedostomía, que ha demostrado una alta eficacia, bajo índice de complicaciones y un resultado estable a largo plazo ${ }^{10-12}$. 日 éxito quirúrgico, definido por la disminución de la diferencia ósea aérea, puede verse afectado por variantes anatómicas del oído medio ${ }^{13}$, las que pueden presentarse hasta en un cuarto de los pacientes que se someten a cirugía, entre las cuales destacan la estrechez del nicho de la ventana oval $(12,3 \%)$, platina flotante $(5,8 \%)$, platina obliterada $(4,7 \%)$, alteraciones en la cadena osicular (3,4\%), sangrado intravestibular (2,4\%) y fijación del martillo $(1 \%)^{14}$. Aros incidentes descritos son el gusher perilinfático que se observa en $0,03 \%$ de los casos $^{15}$ y la arteria estapedial persistente que se describe entre $0,02 \%$ a $0,05 \% 16,17$.

Las alteraciones en la platina han sido descritas como un factor de riesgo para el fracaso quirúrgi$\mathrm{co}^{18}$ y antiguamente algunos autores las conside raban como causa de suspensión de la intervención ${ }^{19}$. La relevancia de estas variantes es que no existe una forma clínica eficaz de predecirlas, por lo que su diagnóstico se realiza durante el procedimiento quirúrgico, y el cirujano otólogo debe estar preparado para enfrentar este tipo de situaciones. Ante estos desafíos se han descrito técnicas quirúrgicas que permiten reducir el riesgo de fracaso y complicaciones. Una de ellas es la realización de una microfenestra en la platina pre vio al retiro de la supraestructura. De este modo si posterior a la extracción de la supraestructura se crea una platina flotante, no represente problema colocar la prótesis en la microfenestra ${ }^{20,21}$. Ara técnica es el fresado de las platinas obliteradas ${ }^{22}$. Lo anterior junto a la experiencia del equipo quirúrgico permiten conseguir resultados satisfactorios, por ejemplo en platinas flotantes $85 \%$ de los pacientes tienen un buen resultado auditivo ${ }^{18}$, mientras que en platinas obliteradas éste se aproxima a $75 \%$ 22,23. 
日 objetivo de este trabajo es demostrar los resultados auditivos postquirúrgicos en un grupo de pacientes con otoesclerosis sometidos a estapedostomía en los cuales se pesquisó la presencia de alteraciones anatómicas en la platina del estribo.

\section{MATERIAL Y MÉTODO}

Se realizó un estudio retrospectivo, en el cual se incluyeron los pacientes con diagnóstico de otosclerosis sometidos a estapedostomía en el Hospital Cínico de la Universidad de Chile entre los años 2000 y 2005. Según los hallazgos anatómicos obtenidos desde el protocolo operatorio se definieron dos grupos, el primero con aquellos pacientes que presentaban una platina definida como complicada (obliterada o flotante), y se utilizó como grupo control aquellos pacientes que presentaban una platina azul.

Todos los pacientes se habían realizado audiometrías tonales puras pre y postoperatorias (tres meses tras intervención) con medición de umbrales de vía aérea, de vía ósea, y diferencia ósea-aérea. Para la expresión de los resultados se utilizaron los valores promedios de las frecuencias $500,1.000,2.000$ y $3.000 \mathrm{~Hz}$.

Se excluyeron de este protocolo aquellos registros clínicos y/o audiológicos incompletos y las cirugías de revisión realizadas.

Se compararon los valores auditivos prome dios de ambos grupos según t Student, considerándose significativo un $p$ menor a 0,05.

\section{RESULTADOS}

Durante el período comprendido entre los años 2000 y 2005 catorce pacientes con otoesclerosis sometidos a estapedostomía presentaban platinas complicadas. Once pacientes (81\%) eran de sexo femenino, con un promedio de edad de 47 años, rango de 33 a 66 años. 日 50\% presentaba enfermedad bilateral y $10 \%$ tenía el antecedente de estapedostomía bilateral. Se consideró como gru- po control a 102 pacientes sometidos a estape dostomía con descripción de platina azul, no encontrándose diferencias en distribución por edad y género.

Dentro del grupo de pacientes con platinas definidas como complicadas $57 \%$ presentaba obliteración de la ventana oval y $43 \%$ platina flotante. Se constataron otros hallazgos anatómicos en el oído medio, en 2 pacientes el nervio facial se encontraba procidente y en 1 se encontraba dehiscente, cifras similares a las encontradas en el grupo con platina azul. No hubo casos en que existiera imposibilidad de completar la cirugía, no se registraron complicaciones postoperatorias y ningún paciente presentó compromiso auditivo sensorioneural.

Los valores audiométricos preoperatorios según el aspecto de la platina se muestran en la Figura 1. Si bien existe una diferencia entre los grupos en cuanto a los umbrales y la diferencia ósea-aérea, ésta no es estadísticamente significativa. Los valores audiométricos postoperatorios según el aspecto de la platina se muestran en la Figura 2, destacando que tanto los valores de umbrales óseo y aéreo, como la diferencia óseoaérea, los promedios son mejores en los pacientes con platina azul, pero esto no constituye una diferencia significativa Los valores comparados en cada uno de los gráficos presentaron una $p$ no significativa.

\section{DISCUSIÓN}

La estapedostomía es un procedimiento quirúrgico altamente efectivo, con un bajo índice de complicaciones y resultado estable a largo plazo. Dentro de las dificultades a las que puede verse enfrentado el cirujano otólogo se encuentran las alteraciones en la platina, las que han sido descritas como un factor de riesgo para el fracaso quirúrgico e, incluso de resolverse de forma apropiada, pueden determinar una peor ganancia auditiva ${ }^{18,20-23}$.

日 manejo de la platina flotante es un desafío, ya que si los intentos por removerla causan mayor daño es posible provocar una hipoacusia sensorioneural. 


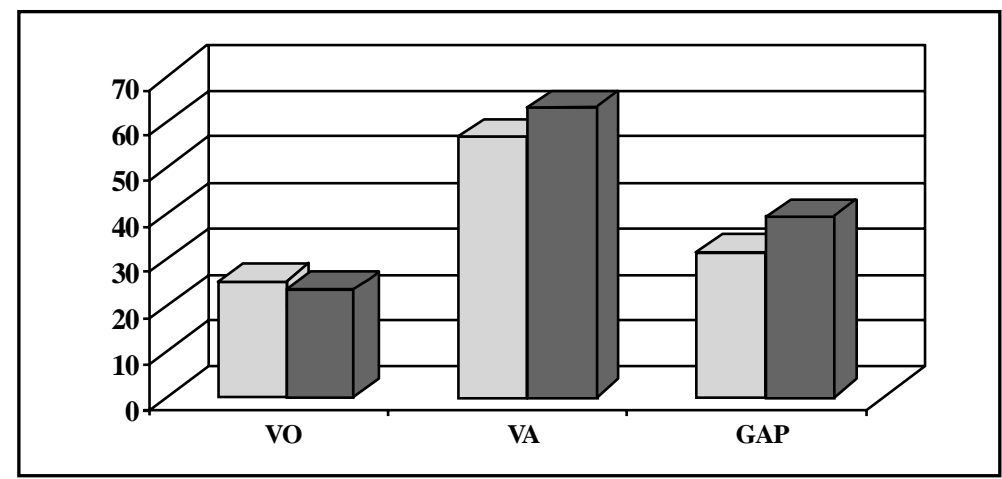

Figura 1. Valores audiométricos preoperatorios según aspecto de platina VO: Umbral vía ósea. VA: Umbral vía aérea. GAP: Diferencia ósea-aérea.

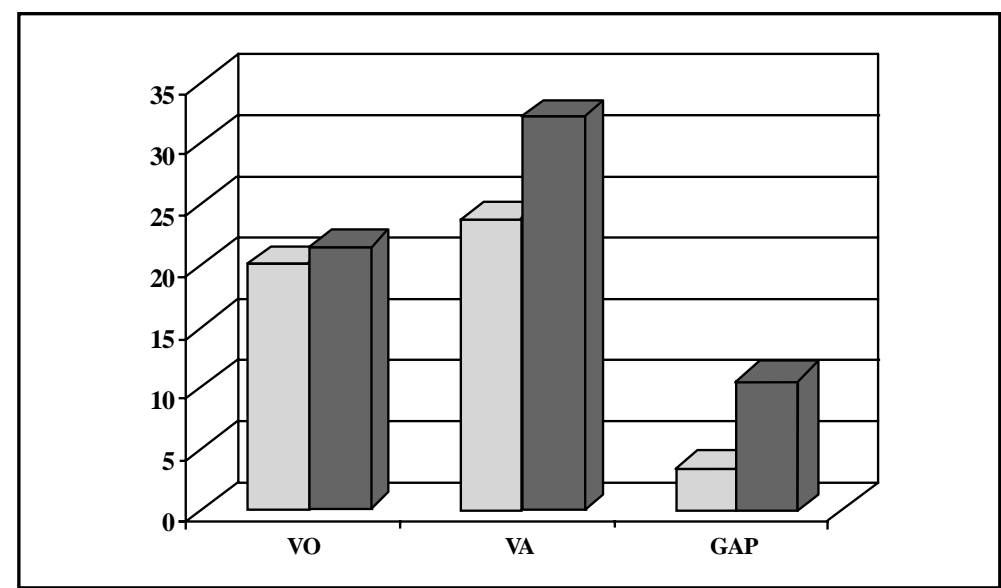

Figura 2. Valores audiométricos postoperatorios según aspecto de platina. VO. Umbral vía ósea VA: Umbral vía aérea. GAP: Diferencia ósea-aérea.

Dada esta posibilidad siempre ( $100 \%$ de los casos) realizamos la platinostomía previo al retiro de la supraestructura, lo que disminuye considerablemente la posibilidad de generar una platina flotante $y$, en caso de que se produzca, permite colocar la prótesis en la platinostomía ya realizada. Esto sería imposible en una platina flotante sin microfenestra

Los primeros reportes de cirugías en platinas obliteradas se acompañaron de hipoacusia sensorioneural ${ }^{24}$ y de reobliteración en $50 \%$ de los $\operatorname{casos}^{25}$. Posteriormente se han demostrado resultados satisfactorios con un lento y cuidadoso fresado de la platina hasta obtener una coloración azul, lo que permite realizar posteriormente la técnica quirúrgica habitual sin inconvenientes. Además esta técnica permite una disminución de la reobliteración, que podría producirse si se crea sólo una microfenestra

La introducción del láser en este tipo de cirugías sería favorable ya que disminuye la posibilidad de trauma, pero no contamos con experiencia con este elemento. Otro punto destacable es que no se utilizó, en nuestra serie, tejido conectivo para sellar la ventana, sin presentarse complicaciones. 
De acuerdo a estas recomendaciones, no hay casos en esta serie en que la cirugía no se pudiera completar de forma satisfactoria. Además, en el seguimiento audiológico postoperatorio la presencia de platinas flotantes u obliteradas no afectó de forma significativa el resultado auditivo, lo que confirma la resolución exitosa de estos desafíos quirúrgicos. Debe destacarse que, además de consideraciones en los pasos quirúrgicos, un factor fundamental es la experiencia del equipo quirúrgico, dado que el manejo prolijo de la técnica y la capacidad de resolver adecuadamente dificultades imprevistas es lo que finalmente permite obtener un resultado exitoso.

\section{BIBLIOGRAFÍA}

1. Causse JR, Causse JB. Otospongiosis as a genetic disease: Early detection, medical management and prevention. Am J Otology 1984; 5: 211-23.

2. SZIKLAI I, KISS JG, RIBARI O. Inhibition of myosin light-chain kinase activity in the organ of Corti by 0.3-5 kilodalton substances of the otosclerotic perilymph. Arch Atorhinolaryngol 1986; 243(4): 229-32.

3. SZIKLAI I. Human otosclerotic bonederived peptide decreases the gain of the electromotility in isolated outer hair cells. Hear Res. 1996;95(1-2):100-7.

4. Bunia J, alsalamei S, Jere R, sittinger $M$, WilmES E BURMESTtR G Antibodies to the minor cartilage collagen type IX in otosclerosis. Am J Otol 1994; 15(2): 222-4.

5. HARRIS JO, WoOlF NK, RYAN AF. A reexamination of experimental type II collagen autoimmunity: middle and inner ear morphology and function. Ann Otol Rhinol Laryngol 1986; 95(2 Pt 1): 176-80.

6. MCKBNNA MJ, MILLS BG Ultrastructural and immunohistochemical evidence of measles virus in active otosclerosis. Acta Otolaryngol 1990; 470(suppl): 130-40.

7. MCKBNNA MJ, MILLS BG Immunohistochemical evidence of measles virus antigens in active otosclerosis. Atolaryngol Head Neck Surg 1989; 101(4): 415-21.

8. HU⿴囗十 MM, GOYCOOLEA MV, PAPARELA MM, OlVERA JA. Otosclerosis: the University of Minnesota temporal bone collection. Aolaryngol Head Neck Surg 1991; 105(3): 396-405.

9. SCHUKNECHT HF, BARBRR W. Histologic variants in otosclerosis. Laryngoscope 1985; 95: 130717.

10. SPANDOW O, SODERBERG O, BOHLIN L. Longterm results in otosclerotic patients operated by stapedectomy or stapedotomy. Scand Audiol 2000; 29(3): 186-90.

11. Lanas A, StotT C, OavarRía C. Otoesclerosis: resultados auditivos de estapedectomía y estapedostomía Rev Aorrinolaringol Cir Cabeza Quello 2002; 62: 115-22.

12. House HP, Hansen MR, Al DakHall AA, House JW. Stapedectomy versus stapedotomy: comparison of results with long-term follow-up. Laryngoscope 2002; 112: 2046-50.

13. KONARSKA A. Abnormalities in the tympanic cavity in otosclerotic patients. Otolaryngol Pol 2005; 59(6): 843-8.

14. Ayache D, Sleman J, Tahuente aN, Elbaz P. Variations and incidents encountered during stapes surgery for otosclerosis. Ann Aolaryngol Chir Cervicofac 1999; 116: 8-14.

15. CAUSSE JB, CAUSSE JR. Eghteen year report on stapedectomy I: Problems of stapedial fixation. Cin Otolaryngol 1980; 5: 49-59.

16. Moreano 日,, Paparella MM, Zilterman D, GOYCOOLEA MV. Prevalence of facial canal deshicence and of persistent stapedial artery in the human middle ear: A report of 1,000 temporal bones. Laryngoscope 1994; 104: 309-20.

17. STEm TN. Vascular anomalies of the middle ear. Laryngoscope 1968; 78: 171-97.

18. G田促 T, KLIMCZAK-GOLAB L, ZBROWSKA-BIESKA D, MAJZH K, WiTKOWSKA M. Results of stapedotomy in the cases of «floating footplate». Otolaryngol Pol 2005; 59: 263-6.

19. CODY DTR, HALLBERG OE, SIMONTON KN. Stape dectomy for otosclerosis: Some causes of failure. Arch Aolaryngol 1967; 85: 184-9. 
20. MANGHAM CA JR. Reducing footplate complications in small fenestra microdrill stapedotomy. Am J Otol 1993; 14: 118-21.

21. GOLABeK W, SZYManSKI M, SIMIEC H, KLOS A. Complications of three methods of stapedectomy. Atolaryngol Pol 2001; 55(6): 593-7.

22. Ayache D, Slgman J, Plouin-Gaudon I, Klap P, EBBAZ P. Obliterative otosclerosis. J Laryngol Otol 1999; 113: 512-4.
23. Cajade Frías J, Labella Caballero T, OrdosgOTía OSORIO H, CASTRO VILAS C, FRADE GONZÁLEZ C. Otosclerosis surgery: hearing results and complications. Acta Atorrinolaringol Esp 2000; 51(3): 199-206.

24. GUILFORD F. Panel on footplate pathology, techniques and prognosis. Arch Atolaryngol 1963; 78: 520-6.

25. HOUSE HP. Footplate surgery in otosclerosis. $J$ Laryngol 1962; 76: 73-86.

Dirección: Dr. Carlos Stott Caro

Av. Santos Dumont 999. Independencia, Santiago

Fax: 56-2-7777338

Email: stott@entelchile.net 